\title{
Successful Pregnancy Outcome with Glioblastoma Multiforme of the Brain
}

Shamim Khandaker

\begin{abstract}
Brain tumors in pregnancy are uncommon occurrences. A 30 -year-old female at 35 weeks of gestation presented with headache, left sided weakness and parasthesias. Brain imaging (MRI) studies revealed a left sided enhancing temporoparietal lesion with perilesional vasogenic edema and mass effect toward right side. She underwent an elective cesarean delivery. Glioblastoma multiforme is a rare diagnosis during pregnancy which may present as neurologic symptoms.
\end{abstract}

Keywords: Pregnancy, Brain tumor, Glioblastoma multiforme.

How to cite this article: Khandaker S. Successful Pregnancy Outcome with Glioblastoma Multiforme of the Brain. J South Asian Feder Menopause Soc 2014;2(1):31-32.

\section{Source of support: Nil}

\section{Conflict of interest: None}

\section{INTRODUCTION}

Brain tumors in pregnancy are rare with an incidence of 15 per 100,000 and previous reports have shown variable outcomes. We present a case of glioblastoma multiforme $(\mathrm{GBM})$ in pregnancy in which the patient delivered a healthy baby with good outcome.

\section{CASE REPORT}

A 30 years old healthy second gravida, presented at 35 weeks' gestation with history of sudden onset of severe headache associated with left sided weakness and blurring of vision in left eye for 1 month. The headache is continuous and associated with vomiting. Physical examination confirmed a glasgow coma scale (GCS) score of 13, unequal pupils, partial cranial nerve III palsy and left hemi paresis. She is normotensive. She has one normal delivery 9 years back.

Magnetic resonance imaging (MRI) showed a large heterogeneously enhancing mass with areas of necrosis in left temporoparietal lobe with perilesional vasogenic edema.

Residential Medical Officer

Department of Obstetrics and Gynecology, North Bengal Medical College, Darjeeling, West Bengal, India

Corresponding Author: Shamim Khandaker, Residential Medical Officer, Department of Obstetrics and Gynecology, North Bengal Medical College, Darjeeling, West Bengal, India, e-mail: shamim_ khandaker@yahoo.co.in
The mass compresses the left lateral ventricle with midline shift toward right side is approximately $15 \mathrm{~mm}$. Right lateral ventricle is dilated. The mass measures $6.6 \times 4.5 \times 4.1 \mathrm{~cm}$ suggest high grade glioma (glioblastoma multiforme) (Fig. 1).

She was transferred to a tertiary center where an obstetric ultrasound showed an appropriately grown fetus. Risks of preterm labor and complications of prematurity were discussed. The diagnosis, treatment options and prognosis of glioblastoma multiforme were discussed with multidisciplinary inputs from neuromedicine, neurosurgeon, obstetrician and therapeutic radiologist. As her fetus reached age of viability; she was planned for elective delivery before appropriate therapeutic measures for brain tumor. She went on to deliver a healthy male baby with a birth weight of $2280 \mathrm{gm}$ via elective lower segment cesarean section (LSCS) and good Apgar scores. Post LSCS she was recovered well and undergone definitive management for her brain treatment.

\section{DISCUSSION}

Brain tumors in pregnancy are relatively rare with an incidence of 15 per $100,000 .{ }^{1}$ Approximately $75 \%$ of cases of intracranial tumors occurring in women of reproductive age presented during pregnancy. This may be explained by an enlargement of the tumor due to fluid retention and accumulation as a result of hormonal changes, vascular engorgement, and hormone related tumor growth, demonstrated by progression of neurologic symptoms during pregnancy and remission postpartum. ${ }^{2}$

Brain tumors in pregnancy can present with a variety of symptoms and signs ranging from mild to severe. Common presenting symptoms include generalized symptoms such as headache, nausea, vomiting, seizures, syncope and cognitive dysfunction which mimic those of pre-eclampsia and eclampsia and hence may result in difficulty or delay in diagnosis. Common focal symptoms include visual, language, motor and sensory disturbances.

Factors like severity and rate of progression of symptoms, gestational age at the time of diagnosis, location and size of the tumor will determine the most appropriate management. A multidisciplinary approach is clearly indicated.

Pharmacological interventions include corticosteroids to reduce intracranial edema and accelerate fetal lung 


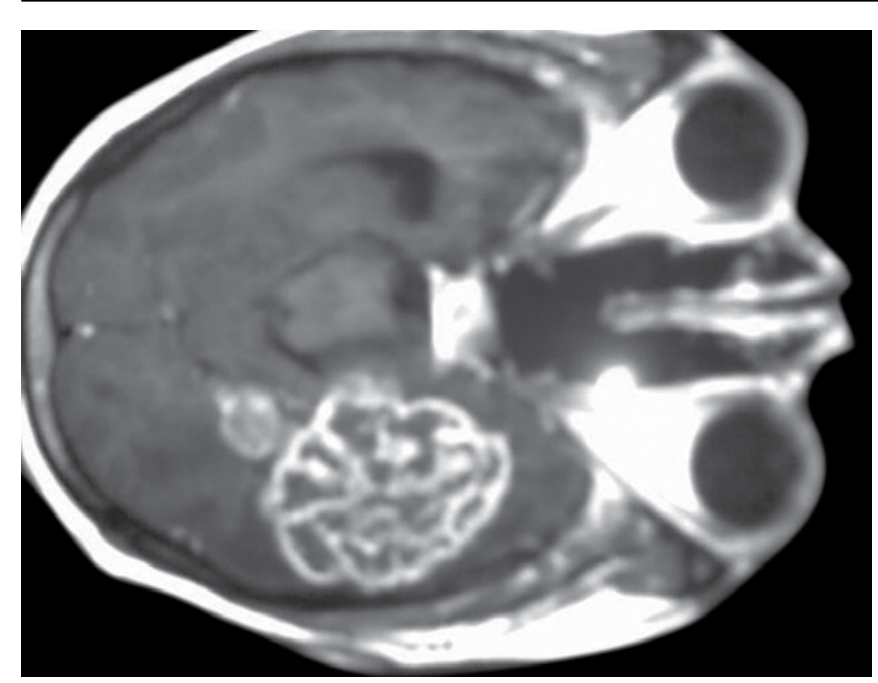

Fig. 1: MRI brain showing mass with areas of necrosis in left temporoparietal lobe, likely to be glioblastoma multiforme

maturity and antiepileptics to control maternal seizures or for prophylaxis. Other forms of treatment include surgical resection, radiation therapy and chemotherapy. ${ }^{3}$

A patient who presents during the first or early second trimesters may be considered for neurosurgery as the fetus is remote from viability. Radiotherapy and image-guided surgery may also be an option after the first trimester. ${ }^{4}$

If a patient presents at or near term, delivery may be performed followed by tumor resection. Delivery should preferably be by LSCS under general anesthesia to reduce the risks of cerebral herniation associated with the downbearing effects in vaginal delivery and placement of an epidural catheter.

Generally, if a patient presents at a time in pregnancy when fetal lung maturity has not been achieved, but is clinically stable, she should be allowed to continue with the pregnancy under close supervision. All risks and benefits associated with continuation or termination of pregnancy, the different treatment modalities, the natural history of the disease and possible outcomes, must be explained.

Brain tumors in pregnancy are medical dilemmas with no standard or routine treatment. The aim of treatment is to minimize both maternal and fetal mortality and morbidity which can be achieved by prolonging pregnancy while alleviating complications from the brain tumor.

\section{REFERENCES}

1. Haas JF, Janisch W, Staneczek W. Newly diagnosed primary intracranial neoplasms in pregnant women: a population-based assessment. J Neurol Neurosurg Psychiatry 1986;49(8):874-880.

2. Isla A, Alvarez F, Gonzalez A, Garcia-Grande A, Perez-Alvarez M, Garcia-Blazquez M. Brain tumor and pregnancy. Obstet Gynecol 1997 Jan;89(1):19-23.

3. Mackenzie AP, Levine G, Garry D, Figueroa R. Glioblastoma multiforme in pregnancy. J Matern Fetal Neonatal Med 2005 Jan;17(1):81-83.

4. Tewari KS, Cappuccini F, Asrat T, Flamm BL, Carpenter SE, Disaia PJ. Obstetric emergencies precipitated by malignant brain tumours. Am J Obstet Gynecol 2000;182(5):1215-1221. 